\title{
eHealth Interventions for Dementia - Using WordPress Plugins as a Flexible Dissemination for Dementia Service Providers
}

\author{
Michael REICHOLD ${ }^{\mathrm{a}, 1}$ Marina SELAU ${ }^{\mathrm{a}}$, Elmar GRAESSEL ${ }^{\mathrm{c}}$, Peter L. \\ KOLOMINSKY-RABAS ${ }^{\mathrm{b}}$ and Hans-Ulrich PROKOSCH ${ }^{\mathrm{a}}$ \\ ${ }^{a}$ Chair of Medical Informatics, Friedrich-Alexander-University Erlangen-Nürnberg \\ (FAU), Germany \\ ${ }^{b}$ Interdisciplinary Center for Health Technology Assessment (HTA) and Public Health \\ (IZPH), Friedrich-Alexander-University Erlangen-Nürnberg (FAU), Germany \\ ${ }^{c}$ Center for Health Services Research in Medicine, Department of Psychiatry and \\ Psychotherapy, University Hospital Erlangen, Friedrich-Alexander-University \\ Erlangen-Nürnberg (FAU), Germany
}

\begin{abstract}
The benefits of eHealth interventions for people with dementia and their informal caregivers have been demonstrated in several studies. In times of contact restrictions, digital solutions have become increasingly important, especially for people with dementia and their mostly elderly caregiving relatives, which are at increased risk of severe illness from COVID-19. As in many other health areas, there is a lack of digital interventions in the dementia landscape that are successfully implemented (i.e., put into practice), especially digital interventions that are scientifically evaluated. Evaluated and proven effective digital interventions exist, but these often do not find their way from research into practice and stay on lowlevel implementation readiness. Within the project digiDEM Bayern, a digital platform with digital services and interventions for people affected by dementia (people with dementia, caregivers, volunteers and interested citizens) is established. As one digital intervention for informal caregivers, the 'Angehörigenampel' (caregivers' traffic-light) was developed, which is able to assess the physical and psychological burden of caregivers. This can help to counteract the health effects of caregiving burden early on before it is too late. The development of the digital intervention as a WordPress-plugin was kept generic so that it can easily be adapted to other languages on further websites. The 'intervention as a plugin' approach demonstrates an easy and flexible way of deploying eHealth interventions to other service providers, especially from other countries. The implementation barriers for other service providers are low enough for them to be able to easily integrate the eHealth intervention on their website, enabling more caregivers to benefit from the disseminated eHealth intervention.
\end{abstract}

${ }^{1}$ Corresponding Author, Michael Reichold, Chair of Medical Informatics Friedrich-AlexanderUniversity Erlangen-Nürnberg, Germany; E-mail: michael.reichold@fau.de. 


\section{Introduction}

COVID-19 has clearly demonstrated the importance of digitalization in all areas of society. Moreover, digital deficiencies have become even more visible in the past months. Particularly for people with dementia and their informal caregivers (usually family members or close relatives who provide unpaid care), access to eHealth interventions is becoming more important than ever [1], especially in view of the fact that the risk factors in developing dementia [2] such as older age, cardiovascular disease, diabetes, chronic respiratory disease, and hypertension are the same that also cause a severe or fatal COVID-19 disease [3].

With 50 million people affected worldwide, dementia is one of the major challenges the healthcare systems are facing. In Germany, 1.6 million people suffer from dementia, about 240,000 of which are in Bavaria (a federal state in Germany) [4]. In 2019, the research project 'Digital Dementia Registry Bavaria - digiDEM Bayern' was launched to improve the situation of people with dementia and their caregiving relatives in Bavaria. digiDEM Bayern is based on the results of the preceding project 'Bavarian Dementia Registry - BayDem' [5-7]. The main objectives of digiDEM Bayern [8] are: First, establishing a digital patient register for the acquisition of long-term data of people with dementia and their caregivers. Second, providing a digital platform with digital interventions and services for people with dementia and cognitive impairments as well as for caregivers, volunteers and interested citizens.

The effectiveness of digital interventions for people with dementia and their family caregivers has been demonstrated in several studies [9-11]. Unfortunately, these interventions are often only available while the research study is funded or have other restrictions such as a foreign language. As a result, these interventions are only accessible to a limited group of people with dementia or caregivers. In 2019, Christie et al. showed that only 2 of 12 interventions initially published in scientific journals are still available to informal caregivers and freely accessible on dementia websites [12]. Among other things, these limitations lead to the fact that the availability of digital interventions in practice is assessed as relatively poor [13].

As one digital intervention, a so-called 'Angehörigenampel' (caregivers' traffic light) was developed for the digiDEM Bayern platform. With the help of the 'Angehörigenampel', informal dementia caregivers can assess their caregiver burden on health, namely on the physical and psychological (emotional) health. Particularly in the early stages of dementia, care for people with dementia is mainly provided by family members serving as informal caregivers [14]. Informal caregiving is associated with poorer physical and mental health outcomes [15], for example, a greater risk of becoming depressed or an increased mortality rate. For these reasons, the informal caregivers are often referred to as the 'hidden secondary patients' [16], who need and deserve protection and guidance [17]. Here, eHealth interventions can improve the situation for informal caregivers, which in turn can also lead to potential benefits for care recipients, i.e., people with dementia [18].

The objective of the study is to demonstrate a feasible method for the dissemination of eHealth interventions using the newly developed intervention 'Angehörigenampel' as an example. By lowering the barriers to a successful implementation, affected people should be able to benefit regardless of research-project and language boundaries. 


\section{Methods}

The scientific basis of the newly developed eHealth intervention 'Angehörigenampel' is a questionnaire containing 10 questions to which answers can be given with 4 predefined answer options. A total score is then calculated from the answers given, which can vary between 0 and 30 points. This self-assessment provides a meaningful assessment of the burden experienced by caregiving relatives in the home care situation and allows classification into a low, moderate and high burden. Specific recommendations for further steps are assigned to these levels of severity. The questionnaire - a short version of the Burden Scale for Family Caregivers (BSFC) [19] - is available in more than 20 languages and has been used across the world for many years. The short version's reliability and validity were evaluated in a scientific study, which measured the burden experienced by 351 informal caregivers in home care [20]. The classification system according to 'traffic light colors' is the second principle that is scientifically verified. Pendergrass et al. reviewed the classification of how the degree of burden relates to the risk of physical psychosomatic complaints [21]. Based on the preliminary scientific work, a specification for the eHealth intervention with functional and non-functional requirements was created by a multi-professional team of digiDEM Bayern.

An easy way to bring digital offerings to those affected is by using a website. From a technical point of view, the basis of the digital platform in digiDEM Bayern is the Content Management System (CMS) WordPress (version 5.6). WordPress supports building a multimedia enriched platform by expanding its basic functions with additional plugins. Plugins are a powerful way to add additional features and services to WordPress using PHP-based scripts. In principle, there are almost no limits to what plugins can do in WordPress [22]. Therefore, the digital intervention 'Angehörigenampel' was developed as a WordPress plugin to be easily integrated into our digiDEM Bayern platform. For this purpose, the scripting language PHP was used in compliance with the WordPress PHP Coding Standards [23]. To allow an easy adaptation to local requirements of other dementia service providers, we kept the plugin as generic as possible. Previous to the publication of the intervention on the website, the technical implementation and functionality of the developed plugin were tested by 5 project members.

\section{Results}

Based on the above-mentioned scientific evidence of Gräßel and Pendergrass, a WordPress plugin was developed in PHP, which allows informal caregivers an assessment of their caregiver burden by answering 10 questions of the short version of the BSFC questionnaire. Depending on the result, the caregivers receive further recommendations to strengthen their physical and mental health.

The developed plugin is available as a zip file and can be easily installed on any WordPress website using the WordPress built-in plugin manager. To ensure an easy and flexible integration of the plugin on any WordPress website page at any place, a shortcode support has been added for the plugin. Shortcodes are commands enclosed by square brackets, which are converted by WordPress into elements of a website. This allows calling a PHP function by adding '[digiDEM_Angehoerigenampel]' in the WordPress page editor. As illustrated in Figure 1, additional regular WordPress content 
such as additional details about the intervention or the scientific evidence can be added before and after the embedded plugin-area [24].

The following four functions were implemented in the plugin 'Angehörigenampel': First, having completed the assessment in the selected language, the caregiver receives a rating of their burden and a corresponding visual indication symbolized by a traffic light (low burden $=$ green light, moderate burden $=$ yellow light, high burden $=$ red light $)$ as well as short advice. To monitor and evaluate the usage of the intervention, the results of the assessment are anonymously stored in a separate table.

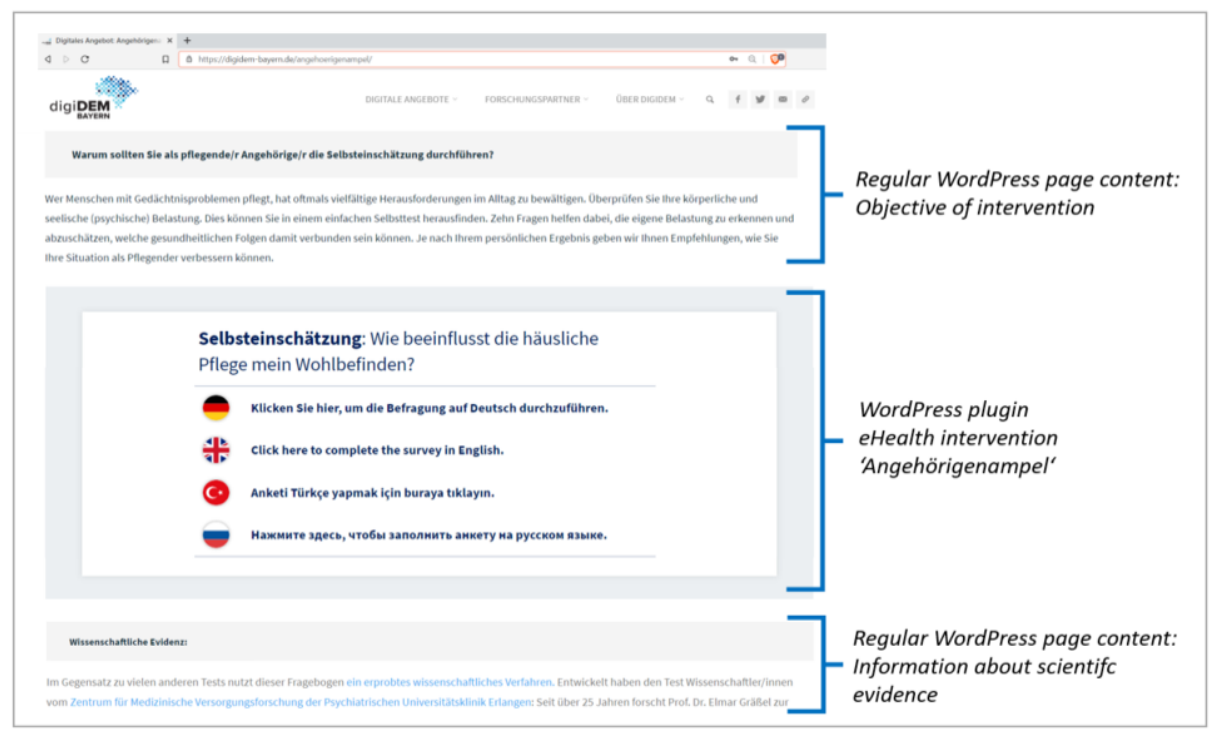

Figure 1. Integration of the plugin 'Angehörigenampel' on the digiDEM Bayern website [24]

Second, the caregiver can download a document corresponding to the identified burden for getting a more detailed assessment and recommended measures for reducing the burden. The recommendations were developed in a panel of experts together with family care advisors. They were then checked for comprehensibility by a group of informal caregivers. Caregivers can take this document with them to their physician or other consulting facilities to take further steps, if necessary. Technically, the document is a structured PDF, generated from a structured HTML and CSS layout file, where individual text areas are combined depending on the individual assessment result. The text modules for the PDF can also be changed directly in WordPress via the plugin's settings.

Third, an optional reminder function has been included after the assessment, which automatically reminds the caregiver to repeat the assessment by email after 6 months. It is recommended to perform the assessment regularly to see how the caregiver burden is developing over time. The period of 6 months was chosen because the progression of dementia caregivers' burden is relatively sluggish [25]. If the caregiver uses the reminder function and enters their email, it will be encrypted (encryption standard: AES-256CBC) together with the date of the assessment in a separate table of the MySQL WordPress database. By using a cron-job, it is checked daily in this table if 6 months have passed since the assessment. If so, the table entry will be decrypted, and a reminder 
email is sent to the caregiver using the wp_mail function provided by WordPress and the table entry is deleted.

Fourth, a feedback section has been included after the assessment to determine how the caregivers accept the eHealth intervention. Here, the caregiver can answer five questions anonymously and on a voluntary basis. Among other things, they can indicate whether the self-assessment accurately reflects their perceived burden of caregiving and whether they would recommend the assessment to other caregivers by means of the Net Promoter Score [26]. The feedback data is stored in an internal MySQL table, and a CSV file can be downloaded for evaluation purposes in the plugin settings. The feedback option of the plugin can be enabled or disabled in the WordPress plugin settings as needed.

To pay attention to the solution's flexibility during the development, the intervention was published initially in four languages (German, English, Turkish, Russian). Due to the multi-language approach, it was possible to immediately test and ensure that an adjustment of the wording or language is possible without programming knowledge for each function. As illustrated in Figure 2, an adaptation to other languages is easily possible via the plugin settings. For this purpose, only the corresponding text modules have to be translated and replaced. If one wants to provide the WordPress plugin to another dementia service provider, the provider only has to upload the plugin to their WordPress installation, activate it and embed it on their website. In the settings of the plugin, they can select whether they want to activate the feedback function. They can also specify which page the reminder email should link to and where the privacy page is, which is referred to in the reminder function.
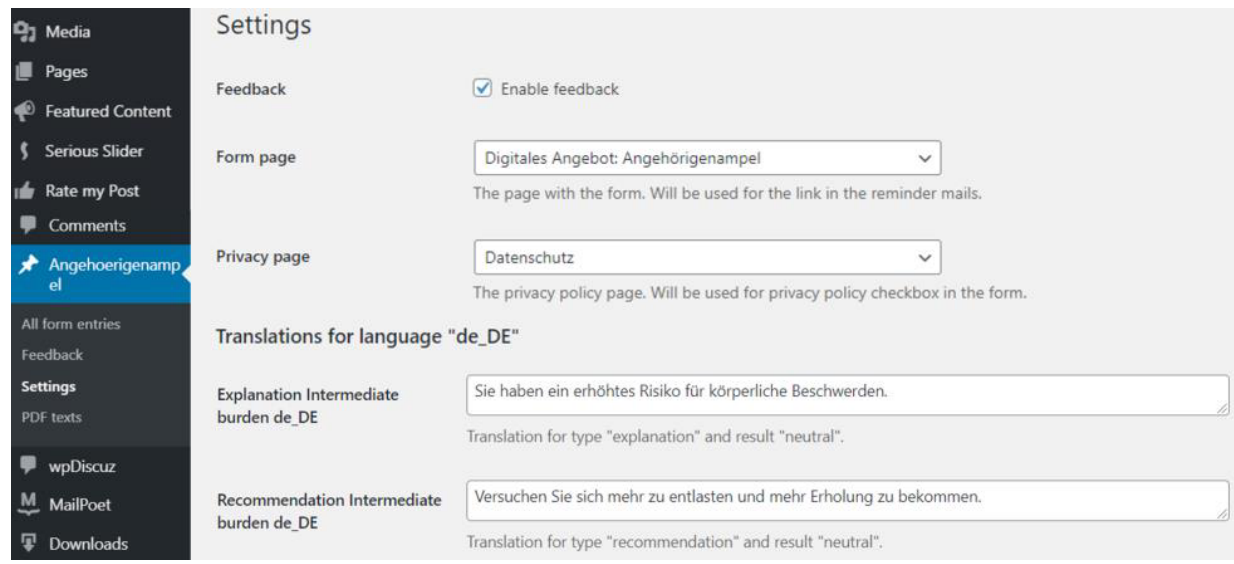

Figure 2. Settings of the WordPress plugin 'Angehörigenampel' of digiDEM Bayern

By deciding to develop the eHealth intervention as a plugin, the following dissemination advantages could be achieved. The website can easily be extended with additional content and functions without having to modify the core of the WordPress system. It also makes it much easier to share the plugin and updates, because one does not have to replace individual pieces of code, which can be very error-prone. 


\section{Discussion}

The objective of this paper was to demonstrate a practical-based example of how an eHealth intervention, which was developed in the context of a dementia research project, can be made available to a broader target group in a simple way.

Talking about digitalization and age, stereotypes always arise about whether the digital way is still the right way to go and whether there are still digital barriers. In contrast to the young generation, older adults are said not to be ready to use the Internet not only technically but also mentally. The latest research on internet use in relation to age shows that older adults are becoming increasingly digital [27]. Corresponding initiatives to overwhelm barriers and further increase eHealth literacy and digital participation are being launched by the policy [28,29]. Particularly for informal caregivers who cannot leave home due to caregiving responsibilities, internet-based interventions provide a cost-effective and flexible way to access interventions from home. Digitalization certainly cannot replace personal contact in every service situation. However, in many cases, digitalization can overcome the limitations of non-digital services such as time, distance and human resources.

Despite evidence of the effectiveness and efficacy of eHealth interventions in numerous studies, there is still a mismatch between the availability of eHealth interventions in research on the one hand and implementation in practice in dementia settings [9]. Gitlin et al. have revealed that for less than 3\% of proven interventions for informal caregivers of people with dementia, the translation from research studies into practice is successful [30]. Although studies demonstrate the effectiveness and benefits of digital interventions, the interventions often remain at a low implementation readiness level [31]. Even though there are already considerations on how to achieve sustainable implementations, these are elaborated only on theoretical examples [32]. The need for digital interventions in practice was reaffirmed in a survey from 2019 among service providers in the dementia care landscape, which found a gap between digital and nondigital dementia services and interventions [13].

Therefore, from the beginning of the development, the focus was on factors enabling a sustainable implementation of the intervention and an adaptation to regional conditions as flexible as possible. To minimize the barriers to implementation, it was ensured that the solution did not demand any major technical requirements in terms of software and hardware and that country-specific adaptations, such as the language, were possible without any programming knowledge. The example of a WordPress plugin was used to show the possibility of developing a generic plugin with a high level of implementation readiness. Country-specific adaptations such as the texts' language can be easily realized via the settings menu of WordPress.

WordPress has by far the largest market share among the top 10 content management systems worldwide, with around 63.9\%. The CMS in second place only had a market share of 5.1\% [33]. The use of WordPress plugins makes it possible to reuse content and solutions easily. The widespread use of WordPress is reflected, for example, in the number of page views of WordPress articles, which in November 2020 was about 25.96 billion [33]. Due to its high penetration, ease of use, low implementation costs, low system requirements, a WordPress-based repository of scientifically validated interventions-plugins in the dementia environment could contribute to the digitalization of other healthcare organizations with similar eHealth needs for their customers. 
As a limitation of the solution as WordPress plugin data privacy should be considered and corresponding protective measures such as anonymization must be taken into account during development. WordPress is the most frequently used CMS in the world, which makes it a valuable target for security vulnerabilities and attacks. Although there are security components and best practices provided by WordPress [34], we store sensitive information only in encrypted or anonymized form in our WordPress environment. A further limitation can be seen in the fact that no data can yet be provided to prove dissemination. However, there are already initiated discussions with Alzheimer's associations in two countries to implement the plugin outside of digiDEM Bayern. Furthermore, there is a request from a palliative care service provider. Due to its easy adaptability, the 'Angehörigenampel' will be made available in an adapted form for informal caregivers of palliative care patients in the next step.

\section{Conclusion}

Digital interventions are a valuable resource to support people with dementia and their informal caregivers. A CMS such as WordPress with its plugins offers a suitable solution in which a sustainable eHealth intervention can be developed rapidly and both disseminated and implemented quickly. The fact that from the beginning of the development the focus was placed on disseminating the eHealth intervention to other providers - together with the fact that it is a customizable WordPress plugin - a very easily adjustable solution was created. This simplifies the installation of eHealth interventions for all interested parties without being restricted by any borders, whether research-project or geographical borders, because dementia does not respect such borders.

\section{Acknowledgments}

The project digiDEM Bayern is funded by the Bavarian State Government as part of the funding initiative 'BAYERN DIGITAL II' (funding code: G42d-G8300-2017/1606-83).

The present work was performed by Michael Reichold in (partial) fulfillment of the requirements for obtaining the degree "Dr. rer. biol. hum." from Faculty of Medicine of the Friedrich-Alexander-University Erlangen-Nürnberg.

\section{Disclosure Statement}

The authors have no conflict of interest to declare.

\section{References}

[1] H. Wang, T. Li, P. Barbarino, S. Gauthier, H. Brodaty, J.L. Molinuevo, H. Xie, Y. Sun, E. Yu, Y. Tang, W. Weidner, and X. Yu, Dementia care during COVID-19, Lancet. 395 (2020) 1190-1191. doi:10.1016/S0140-6736(20)30755-8. 
[2] S. Norton, F.E. Matthews, D.E. Barnes, K. Yaffe, and C. Brayne, Potential for primary prevention of Alzheimer's disease: an analysis of population-based data, Lancet Neurol. 13 (2014) 788-794. doi:10.1016/S1474-4422(14)70136-X.

[3] Z. Wu, and J.M. McGoogan, Characteristics of and Important Lessons From the Coronavirus Disease 2019 (COVID-19) Outbreak in China: Summary of a Report of 72314 Cases From the Chinese Center for Disease Control and Prevention, JAMA. 323 (2020) 1239-1242. doi:10.1001/jama.2020.2648.

[4] Bayerisches Landesamt für Gesundheit und Lebensmittelsicherheit, Gesundheitsreport Bayern: 2/2019 - Update Demenzerkrankungen, (2019). https://www.lgl.bayern.de/publikationen/doc/gesundheitsreport_2_2019.pdf (accessed January 8, 2020).

[5] L. Karrer, N. Dietzel, F. Wolff, A. Kratzer, M. Hess, E. Gräßel, and P. Kolominsky-Rabas, [Use of Outpatient Care Services by People with Dementia: Results of the Bavarian Dementia Survey (BayDem)], Gesundheitswesen. 82 (2020) 40-49. doi:10.1055/a-1071-7851.

[6] F. Wolff, N. Dietzel, L. Karrer, A. Kratzer, M. Hess, E. Gräßel, and P. Kolominsky-Rabas, Zeitgerechte Diagnosestellung bei Menschen mit Demenz: der Bayerische Demenz Survey (BayDem), Gesundheitswesen. 82 (2020) 23-29. doi:10.1055/a-1031-9559.

[7] N. Dietzel, L. Karrer, F. Wolff, A. Kratzer, M. Hess, E. Gräßel, and P. Kolominsky-Rabas, [Predictors of Caregiver Burden in Dementia: Results of the Bavarian Dementia Survey (BayDem)], Gesundheitswesen. 82 (2020) 30-39. doi:10.1055/a-1071-7886.

[8] N. Dietzel, L. Kürten, M. Reichold, L. Köhler, C. Chmelirsch, K. Seebahn, M. Hladik, S. Meuer, A. Kirchner, K. Holm, M. Selau, M. Wendel, J. Trinkwalter, H.-U. Prokosch, E. Gräßel, P.L. KolominskyRabas, and (in press), The Digital Dementia Registry Bavaria - digiDEM Bayern: study protocol for a multicentre, prospective, longitudinal register study, BMJ Open. (2021).

[9] H.L. Christie, S.L. Bartels, L.M.M. Boots, H.J. Tange, F.R.J. Verhey, and M.E. de Vugt, A systematic review on the implementation of eHealth interventions for informal caregivers of people with dementia, Internet Interventions. 13 (2018) 51-59. doi:10.1016/j.invent.2018.07.002.

[10] E. Parra-Vidales, F. Soto-Pérez, M.V. Perea-Bartolomé, M.A. Franco-Martín, and J.L. Muñoz-Sánchez, Online interventions for caregivers of people with dementia: a systematic review, Actas Esp Psiquiatr. 45 (2017) 116-126.

[11] J.L. Scott, S. Dawkins, M.G. Quinn, K. Sanderson, K.-E.J. Elliott, C. Stirling, B. Schüz, and A. Robinson, Caring for the carer: a systematic review of pure technology-based cognitive behavioral therapy (TB-CBT) interventions for dementia carers, Aging Ment Health. 20 (2016) 793-803. doi:10.1080/13607863.2015.1040724.

[12] H.L. Christie, J.L. Martin, J. Connor, H.J. Tange, F.R.J. Verhey, M.E. de Vugt, and M. Orrell, eHealth interventions to support caregivers of people with dementia may be proven effective, but are they implementation-ready?, Internet Interventions. 18 (2019) 100260. doi:10.1016/j.invent.2019.100260.

[13] M. Reichold, N. Dietzel, L. Karrer, E. Graessel, P.L. Kolominsky-Rabas, and H.-U. Prokosch, Stakeholder Perspectives on the Key Components of a Digital Service Platform Supporting Dementia digiDEM Bayern, Stud Health Technol Inform. 271 (2020) 224-231. doi:10.3233/SHTI200100.

[14] Statistisches Bundesamt, Pflege im Rahmen der Pflegeversicherung - Deutschlandergebnisse, (2017). https://www.destatis.de/DE/Themen/Gesellschaft-

Umwelt/Gesundheit/Pflege/Publikationen/Downloads-Pflege/pflege-deutschlandergebnisse5224001179004.pdf?_ blob=publicationFile (accessed January 8, 2020).

[15] J. Bom, P. Bakx, F. Schut, and E. van Doorslaer, The Impact of Informal Caregiving for Older Adults on the Health of Various Types of Caregivers: A Systematic Review, The Gerontologist. (2018). doi:10.1093/geront/gny137.

[16] H. Brodaty, and M. Donkin, Family caregivers of people with dementia, Dialogues Clin Neurosci. 11 (2009) 217-228.

[17] S.C. Reinhard, B. Given, N.H. Petlick, and A. Bemis, Supporting Family Caregivers in Providing Care, in: R.G. Hughes (Ed.), Patient Safety and Quality: An Evidence-Based Handbook for Nurses, Agency for Healthcare Research and Quality (US), Rockville (MD), 2008. http://www.ncbi.nlm.nih.gov/books/NBK2665/ (accessed December 19, 2020).

[18] M. Leng, Y. Zhao, H. Xiao, C. Li, and Z. Wang, Internet-Based Supportive Interventions for Family Caregivers of People With Dementia: Systematic Review and Meta-Analysis, Journal of Medical Internet Research. 22 (2020) e19468. doi:10.2196/19468.

[19] E. Graessel, R. Chiu, and R. Oliver, Development and validation of the Burden Scale for Family Caregivers (BSFC), Comprehensive Rehabilitation and Mental Health Services. (2003) Toronto (Canada).

[20] E. Graessel, H. Berth, T. Lichte, and H. Grau, Subjective caregiver burden: validity of the 10-item short version of the Burden Scale for Family Caregivers BSFC-s, BMC Geriatr. 14 (2014) 23. doi:10.1186/1471-2318-14-23. 
[21] A. Pendergrass, C. Malnis, U. Graf, S. Engel, and E. Graessel, Screening for caregivers at risk: Extended validation of the short version of the Burden Scale for Family Caregivers (BSFC-s) with a valid classification system for caregivers caring for an older person at home, BMC Health Serv Res. 18 (2018) 229. doi:10.1186/s12913-018-3047-4.

[22] B. Williams, J. Tadlock, and J.J. Jacoby, Professional WordPress plugin development, 2020.

[23] PHP Coding Standards, Make WordPress Core. (2013). https://make.wordpress.org/core/handbook/best-practices/coding-standards/php/ (accessed December 16, 2020).

[24] Digitales Angebot: Angehörigenampel, digiDEM Bayern. (n.d.). https://digidembayern.de/angehoerigenampel/ (accessed December 20, 2020).

[25] M.H. Connors, K. Seeher, A. Teixeira-Pinto, M. Woodward, D. Ames, and H. Brodaty, Dementia and caregiver burden: A three-year longitudinal study, Int J Geriatr Psychiatry. 35 (2020) 250-258. doi: $10.1002 / \mathrm{gps} .5244$.

[26] D.F. Hamilton, J.V. Lane, P. Gaston, J.T. Patton, D.J. MacDonald, A.H.R.W. Simpson, and C.R. Howie, Assessing treatment outcomes using a single question: the Net Promoter Score, The Bone \& Joint Journal. 96-B (2014) 622-628. doi:10.1302/0301-620X.96B5.32434.

[27] O. Huxhold, and K. Otte, Internetzugang und Internetnutzung in der zweiten Lebenshälfte. (dza-aktuell: Deutscher Alterssurvey, 01/2019), Deutsches Zentrum für Altersfragen, Berlin, 2019.

[28] Bundesministerium für Familie, Senioren, Frauen und Jugend Referat Öffentlichkeitsarbeit, Achter Altersbericht - Ältere Menschen und Digitalisierung, (2020). https://www.achteraltersbericht.de/fileadmin/altersbericht/pdf/aktive_PDF_Altersbericht_DT-Drucksache.pdf.

[29] A. Efthymiou, I. Menikou, A. Stylianides, N. Geronikola, I. Ilieva, I. Kancheva, M. Tagliani, R. Seneca, B. Mintus, P. Sakka, and E. Papastavrou, eHealth Literacy Training Among Carers of Older People and People with Dementia: A Modified Delphi Survey, Stud Health Technol Inform. 272 (2020) 119-122. doi:10.3233/SHTI200508.

[30] L.N. Gitlin, K. Marx, I.H. Stanley, and N. Hodgson, Translating Evidence-Based Dementia Caregiving Interventions into Practice: State-of-the-Science and Next Steps, The Gerontologist. 55 (2015) 210-226. doi:10.1093/geront/gnu123.

[31] J.V. Lacey, and K.E. Savage, $50 \%$ Response rates: half-empty, or half-full?, Cancer Causes Control. 27 (2016) 805-808. doi:10.1007/s10552-016-0748-z.

[32] H.L. Christie, L.M.M. Boots, K. Peetoom, H.J. Tange, F.R.J. Verhey, and M.E. de Vugt, Developing a Plan for the Sustainable Implementation of an Electronic Health Intervention (Partner in Balance) to Support Caregivers of People With Dementia: Case Study, JMIR Aging. 3 (2020) e18624. doi:10.2196/18624.

[33] Content-Management-Systeme (CMS) - Marktanteile 2020, Statista. (n.d.). https://de.statista.com/statistik/daten/studie/320670/umfrage/marktanteile-der-content-managementsysteme-cms-weltweit/ (accessed December 15, 2020).

[34] WordPress is Secure, WordPress.Org. (2018). https://wordpress.org/about/security/ (accessed December 17, 2020). 